\title{
Nonlinear Stability and D-Convergence of Additive Runge-Kutta Methods for Multidelay-Integro-Differential Equations
}

\author{
Haiyan Yuan, ${ }^{1,2}$ Jingjun Zhao, ${ }^{1}$ and Yang $X u^{1}$ \\ ${ }^{1}$ Department of Mathematics, Harbin Institute of Technology, Harbin 150001, China \\ ${ }^{2}$ Department of Mathematics, Heilongjiang Institute of Technology, Harbin 150050, China
}

Correspondence should be addressed to Jingjun Zhao, hit_zjj@hit.edu.cn

Received 30 December 2011; Accepted 19 February 2012

Academic Editor: Muhammad Aslam Noor

Copyright (c) 2012 Haiyan Yuan et al. This is an open access article distributed under the Creative Commons Attribution License, which permits unrestricted use, distribution, and reproduction in any medium, provided the original work is properly cited.

This paper is devoted to the stability and convergence analysis of the Additive Runge-Kutta methods with the Lagrangian interpolation (ARKLMs) for the numerical solution of multidelayintegro-differential equations (MDIDEs). GDN-stability and D-convergence are introduced and proved. It is shown that strongly algebraically stability gives D-convergence, DA- DAS- and ASIstability give GDN-stability. A numerical example is given to illustrate the theoretical results.

\section{Introduction}

Delay differential equations arise in a variety of fields as biology, economy, control theory, electrodynamics (see, e.g., [1-5]). When considering the applicability of numerical methods for the solution of DDEs, it is necessary to analyze the stability of the numerical methods. In the last three decades, many works had dealt with these problems (see, e.g., [6]). For the case of nonlinear delay differential equations, this kind of methodology had been first introduced by Torelli [7] and then developed by [8-12].

In this paper, we consider the following nonlinear multidelay-integro-differential equations (MDIDEs) with $m$ delays:

$$
\begin{aligned}
y^{\prime}(t)= & f^{[1]}\left(t, y(t), y\left(t-\tau_{1}\right), \int_{t-\tau_{1}}^{t} g^{[1]}(t, s, y(s)) d s\right) \\
& +f^{[2]}\left(t, y(t), y\left(t-\tau_{2}\right), \int_{t-\tau_{2}}^{t} g^{[2]}(t, s, y(s)) d s\right)
\end{aligned}
$$




$$
\begin{aligned}
& +\cdots+f^{[m]}\left(t, y(t), y\left(t-\tau_{m}\right), \int_{t-\tau_{2}}^{t} g^{[m]}(t, s, y(s)) d s\right), \quad t \in\left[t_{0}, T\right], \\
y(t)= & \varphi(t), \quad t \in\left[t_{0}-\tau, t_{0}\right],
\end{aligned}
$$

where $\tau_{1} \leq \tau_{2} \leq \cdots \leq \tau_{m}=\tau, f^{[v]}:\left[t_{0}, T\right] \times C^{N} \times C^{N} \times C^{N} \rightarrow C^{N}, g^{[v]}:\left[t_{0}, T\right] \times C^{N} \times C^{N} \rightarrow$ $C^{N} v=1,2, \ldots, m$, and $\varphi:\left[t_{0}-\tau, t_{0}\right] \rightarrow C^{N}$ are continuous functions such that (1.1) has a unique solution. Moreover, we assume that there exist some inner product $\langle\cdot, \cdot\rangle$ and the induced norm $\|\cdot\|$ such that

$$
\begin{aligned}
& \operatorname{Re}\left\langle f^{[v]}\left(t, y_{1}, u_{1}, w_{1}\right)-f^{[v]}\left(t, y_{2}, u_{2}, w_{2}\right), y_{1}-y_{2}\right\rangle \\
& \quad \leq \alpha_{v}\left\|y_{1}-y_{2}\right\|^{2}+\beta_{v}\left\|u_{1}-u_{2}\right\|^{2}+\sigma_{v}\left\|w_{1}-w_{2}\right\|^{2}, \quad v=1,2, \ldots, m, t \geq t_{0}, \\
& \left\|f^{[v]}\left(t, y, u_{1}, w\right)-f^{[v]}\left(t, y, u_{2}, w\right)\right\| \leq r_{v}\left\|u_{1}-u_{2}\right\|, \\
& \left\|g^{[v]}\left(t, s, w_{1}\right)-g^{[v]}\left(t, s, w_{2}\right)\right\| \leq \tilde{r}_{v}\left\|w_{1}-w_{2}\right\|, \quad(t, s) \in D
\end{aligned}
$$

for all $t \in\left[t_{0}, T\right]$, for all $y, y_{1}, y_{2}, u, u_{1}, u_{2}, w, w_{1}, w_{2} \in C^{N},\left(-\alpha_{v}\right), \beta_{v}, \sigma_{v}, r_{v}, \tilde{r}_{v}$ are all nonnegative constants. Throughout this paper, we assume that the problem (1.1) has unique exact solution $y(t)$. Space discretization of some time-dependent delay partial differential equations give rises to such delay differential equations containing additive terms with different stiffness properties. In these situations, additive Runge-Kutta (ARK) methods are used. Some recent works about ARK can refer to [13, 14]. For the additive MDIDEs (1.1), similar to the proof of Theorem 2.1 in [7], it is straightforward to prove that under the conditions (1.2) (1.4), the analytic solutions satisfy

$$
\|y(t)-z(t)\| \leq \max _{t_{0}-\tau \leq t \leq t_{0}}\|\varphi(t)-\psi(t)\|
$$

where $z(t)$ is the solution of the perturbed problem to (1.1).

To demand the discrete numerical solutions to preserve the convergence properties of the analytic solutions, Torelli [7] introduced a concept of RN-, GRN-stability for numerical methods applied to dissipative nonlinear systems of DDEs such as (1.1) when $g^{[v]}(t, s, y(s))=$ $0, v=1,2, \ldots, m$, which is the straightforward generalization of the well-known concept of BN-stability of numerical methods with respect to dissipative systems of ODEs (see also [9]). More recently, one has noticed a growing interesting the analysis of delay integro-differential equations (DIDEs). This type of equations have been investigated in various fields, such as mathematical biology and control theory (see [15-17]). The theory of computational methods for delay integro-differential equations (DIDEs) has been studied by many authors, and a great deal of interesting results have been obtained (see [18-22]). Koto [23] dealt with the linear stability of Runge-Kutta (RK) methods for systems of DIDEs; Huang and Vandewalle [24] gave sufficient and necessary stability conditions for exact and discrete solutions of linear Scalar DIDEs. However, little attention has been paid to nonlinear multidelay-integrodifferential equations (MDIDEs). 
So, the aim of this paper is the study of stability and convergence properties for ARK methods when they are applied to nonlinear multidelay-integro-differential equations (MDIDEs) with $m$ delays.

\section{The GDN-Stability of the Additive Runge-Kutta Methods}

An additive Runge-Kutta method with the Lagrangian interpolation (ARKLM) of $s$ stages and $m$ levels can be organized in the Butcher tableau:

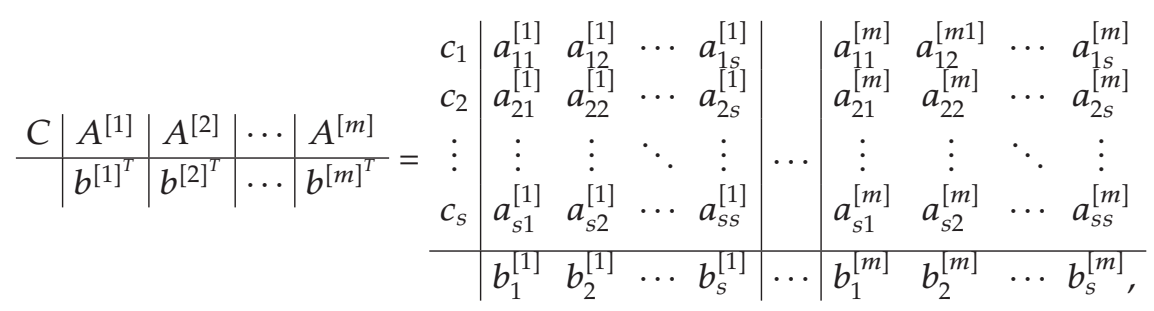

where $C=\left[c_{1}, c_{2}, \ldots, c_{s}\right]^{T}, b^{[v]}=\left[b_{1}^{[v]}, b_{2}^{[v]}, \ldots, b_{s}^{[v]}\right]$, and $A^{[v]}=\left(a_{i j}^{[v]}\right)_{i, j=1}^{S}$.

The adoption of the method (2.1) for solving the problem (1.1) leads to

$$
\begin{aligned}
& y_{n+1}=y_{n}+h \sum_{v=1}^{m} \sum_{j=1}^{s} b_{j}^{[v]} f^{[v]}\left(t_{j}^{(n)}, y_{j}^{(n)}, \tilde{y}_{j}^{[v](n)}, w_{j}^{[v](n)}\right), \\
& y_{i}^{(n)}=y_{n}+h \sum_{v=1}^{m} \sum_{j=1}^{s} a_{i j}^{[v]} f^{[v]}\left(t_{j}^{(n)}, y_{j}^{(n)}, \tilde{y}_{j}^{[v](n)}, w_{j}^{[v](n)}\right),
\end{aligned}
$$

where $t_{n}=t_{0}+n h, t_{j}^{(n)}=t_{n}+c_{j} h, y_{n}$, and $y_{j}^{(n)}, \tilde{y}_{j}^{[v](n)}$ are approximations to the analytic solution $y\left(t_{n}\right), y\left(t_{n}+c_{j} h\right), y\left(t_{n}+c_{j} h-\tau_{v}\right)$ of $(1.1)$, respectively, and the argument $\tilde{y}_{j}^{[v](n)}$ is determined by

$$
\tilde{y}_{j}^{[v](n)}= \begin{cases}\varphi\left(t_{n}+c_{j} h-\tau_{v}\right) & t_{n}+c_{j} h-\tau_{v} \leq 0 \\ \sum_{P_{v}=-d}^{r} L_{P_{v}}\left(\delta_{v}\right) y_{j}^{\left(n-m_{v}+P_{v}\right)} & t_{n}+c_{j} h-\tau_{v}>0\end{cases}
$$

with $\tau_{v}=\left(m_{v}-\delta_{v}\right) h, \delta_{v} \in[0,1)$, integer $m_{v} \geq r+1, r, d \geq 0$, and

$$
L_{P_{v}}\left(\delta_{v}\right)=\prod_{\substack{k=-d \\ k \neq P_{v}}}^{r}\left(\frac{\delta_{v}-k}{P_{v}-k}\right), \quad P_{v}=-d,-d+1, \ldots, r .
$$


We assume $m_{v} \geq r+1$ is to guarantee that no (unknown) values $y_{j}^{(i)}$ with $i \geq n$ are used in the interpolation procedure

$$
w_{j}^{[v](n)} \text { is an approximation to } w\left(t_{j}^{(n)}\right):=\int_{t_{j}^{\left(n-m_{v}\right)}}^{t_{j}^{(n)}} g^{[v]}\left(t_{j}^{(n)}, s, y(s)\right) d s,
$$

which can be computed by a appropriate compound quadrature rule:

$$
w_{j}^{[v](n)}=h \sum_{q=0}^{m_{v}} d_{q} g^{[v]}\left(t_{j}^{(n)}, t_{j}^{(n-q)}, y_{j}^{(n-q)}\right), \quad v=1,2, \ldots, m, j=1,2, \ldots, s .
$$

As for the quadrature rule (2.6), we usually adopt the compound trapezoidal rule, the compound Simpsons rule or the compound Newton-Cotes rule, and so forth according to the requirement of the convergence of the method (see [19]) and denote $M=\max _{1 \leq v \leq m}\left\{m_{v}\right\}$ and $\eta=\max _{1 \leq v \leq m}\left\{\eta_{v}\right\}$ with $\eta_{v}$ satisfing $\sum_{q=0}^{m_{v}}\left|d_{q}\right|<\eta_{v}, v=1,2, \ldots, m$.

In addition, we always put $y_{j}^{(n)}=\varphi\left(t_{n}+c_{j} h\right), y_{n}=\varphi\left(t_{n}\right)$ whenever $n \leq 0$.

In order to write (2.2), (2.3), (2.5), and (2.6) in a more compact way, we introduce some notations. The $N \times N$ identity matrix will be denoted by $I_{N}, e=(1,1, \ldots, 1)^{T} \in R^{S}, \widetilde{G}=G \otimes I_{N}$ is the Kronecker product of matrix $G$ and $I_{N}$. For $u=\left(u_{1}, u_{2}, \ldots, u_{s}\right)^{T}, v=\left(v_{1}, v_{2}, \ldots, v_{s}\right)^{T} \in$ $C^{N S}$, we define the inner product and the induced norm in $C^{N S}$ as follows:

$$
\langle u, v\rangle=\sum_{i=1}^{s}\left\langle u_{i}, v_{i}\right\rangle, \quad\|u\|=\sqrt{\sum_{i=1}^{s}\left\|u_{i}\right\|^{2}}
$$

Moreover, we also adopt that

$$
\begin{gathered}
y^{(n)}=\left[\begin{array}{c}
y_{1}^{(n)} \\
y_{2}^{(n)} \\
\vdots \\
y_{s}^{(n)}
\end{array}\right], \quad \tilde{y}^{[v](n)}=\left[\begin{array}{c}
\tilde{y}_{1}^{[v](n)} \\
\tilde{y}_{2}^{[v](n)} \\
\vdots \\
\tilde{y}_{s}^{[v](n)}
\end{array}\right], \quad w^{[v](n)}=\left[\begin{array}{c}
w_{1}^{[v](n)} \\
w_{2}^{[v](n)} \\
\vdots \\
w_{s}^{[v](n)}
\end{array}\right], \quad T^{(n)}=\left[\begin{array}{c}
t_{1}^{(n)} \\
t_{2}^{(n)} \\
\vdots \\
t_{s}^{(n)}
\end{array}\right], \\
f^{[v]}\left(T^{(n)}, y^{(n)}, \tilde{y}^{[v](n)}, w^{[v](n)}\right)=\left[\begin{array}{c}
f^{[v]}\left(t_{1}^{(n)}, y_{1}^{(n)}, \tilde{y}_{1}^{[v](n)}, w_{1}^{[v](n)}\right) \\
f^{[v]}\left(t_{2}^{(n)}, y_{2}^{(n)}, \tilde{y}_{2}^{[v](n)}, w_{2}^{[v](n)}\right) \\
\vdots \\
f^{[v]}\left(t_{s}^{(n)}, y_{s}^{(n)}, \tilde{y}_{s}^{[v](n)}, w_{s}^{[v](n)}\right)
\end{array}\right] .
\end{gathered}
$$


With the above notation, method (2.2),(2.3), (2.5), and (2.6) can be written as

$$
\begin{gathered}
y_{n+1}=y_{n}+h \sum_{v=1}^{m} \tilde{b}^{[v]^{T}} f^{[v]}\left(T^{(n)}, y^{(n)}, \tilde{y}^{[v](n)}, w^{[v](n)}\right), \\
y^{(n)}=\tilde{e} y_{n}+h \sum_{v=1}^{m} \tilde{A}^{[v]} f^{[v]}\left(T^{(n)}, y^{(n)}, \tilde{y}^{[v](n)}, w^{[v](n)}\right), \\
\tilde{y}_{j}^{[v](n)}= \begin{cases}\tilde{e} \varphi\left(t_{n}+c_{j} h-\tau_{v}\right), & t_{n}+c_{j} h-\tau_{v} \leq t_{0}, \\
\sum_{P_{v}=-d}^{r} L_{P_{v}}\left(\delta_{v}\right) y_{j}^{\left(n-m_{v}+P_{v}\right)}, & t_{n}+c_{j} h-\tau_{v}>t_{0},\end{cases} \\
w_{j}^{[v](n)}=h \sum_{q=0}^{m_{v}} d_{q} g^{[v]}\left(t_{n}+c_{j} h, t_{n-q}+c_{j} h, y_{j}^{(n-q)}\right) .
\end{gathered}
$$

In 1997, Zhang and Zhou [25] introduced the extension of RN-stability to GDN-stability as follows.

Definition 2.1. An ARKLM (2.1) for DDEs is called GDN-stable if, numerical approximations $y_{n}$ and $z_{n}$ to the solution of (1.1) and its perturbed problem, respectively, satisfy

$$
\left\|y_{n}-z_{n}\right\| \leq C \max _{t_{0}-\tau \leq t<t_{0}}\|\varphi(t)-\psi(t)\|, \quad n \geq 0
$$

where constant $C>0$ depends only on the method, the parameter $\alpha_{v}, \beta_{v}, \sigma_{v}, r_{v}, \tilde{r}_{v}$, and the interval length $T-t_{0}, \psi(t)$ is the initial function to the perturbed problem of (1.1).

Definition 2.2. An ARKLM (2.1) is called strongly algebraically stable if matrices $M_{\gamma \mu}$ are nonnegative definite, where

$$
M_{\gamma \mu}=B^{[\gamma]} A^{[\mu]}+A^{[\gamma]^{T}} B^{[\mu]}-b^{[\gamma]} b^{[\mu]^{T}}, \quad B^{[\gamma]}=\operatorname{diag}\left(b_{1}^{[\gamma]}, b_{2}^{[\gamma]}, \ldots, b_{s}^{[\gamma]}\right)
$$

for $\mu, \gamma=1,2, \ldots, m$.

Let

$$
\begin{gathered}
\left\{y_{n}, y_{j}^{(n)}, \tilde{y}_{j}^{[1](n)}, \tilde{y}_{j}^{[2](n)}, \ldots, \tilde{y}_{j}^{[m](n)}, w_{j}^{[1](n)}, w_{j}^{[2](n)}, \ldots, w_{j}^{[m](n)}\right\}_{j=1}^{s} \\
\left\{z_{n}, z_{j}^{(n)}, \tilde{z}_{j}^{[1](n)}, \tilde{z}_{j}^{[2](n)}, \ldots, \widetilde{z}_{j}^{[m](n)}, \widehat{w}_{j}^{[1](n)}, \widehat{w}_{j}^{[2](n)}, \ldots, \widehat{w}_{j}^{[m](n)}\right\}_{j=1}^{s}
\end{gathered}
$$

be two sequences of approximations to problems (1.1) and its perturbed problem, respectively. From method (2.1) with the same step size $h$, and write

$$
\begin{array}{r}
U_{i}^{(n)}=y_{i}^{(n)}-z_{i}^{(n)}, \quad \tilde{U}_{i}^{[v](n)}=\tilde{y}_{i}^{[v](n)}-\widetilde{z}_{i}^{[v](n)}, \quad U_{0}^{(n)}=y_{n}-z_{n}, \\
Q_{i}^{[v](n)}=h\left[f^{[v]}\left(t_{i}^{(n)}, y_{i}^{(n)}, \tilde{y}_{i}^{[v](n)}, w_{i}^{[v](n)}\right)-f^{[v]}\left(t_{i}^{(n)}, z_{i}^{(n)}, \widetilde{z}_{i}^{[v](n)}, \widehat{w}_{i}^{[v](n)}\right)\right], \\
i=1,2, \ldots, s, \quad v=1,2, \ldots, m .
\end{array}
$$


Then (2.2) and (2.3) read

$$
\begin{aligned}
& U_{0}^{(n+1)}=U_{0}^{(n)}+\sum_{v=1}^{m} \sum_{j=1}^{s} b_{j}^{[v]} Q_{j}^{[v](n)}, \\
& U_{i}^{(n)}=U_{0}^{(n)}+\sum_{v=1}^{m} \sum_{j=1}^{s} a_{i j}^{[v]} Q_{j}^{[v](n)} .
\end{aligned}
$$

Our main results about GDN-stability are contained in the following theorem.

Theorem 2.3. Assume ARK method (2.2) is strongly algebraically stable, and then the corresponding ARKLM (2.1) is GDN-stable, and satisfies

$$
\left\|y_{n}-z_{n}\right\| \leq C \max _{t_{0}-\tau \leq t \leq t_{0}}\|\varphi(t)-\psi(t)\|^{2}, \quad n \geq 0
$$

where

$$
C=\exp \left[6\left(T-t_{0}\right) m s \sum_{v=1}^{m} \beta_{v} L_{v}^{2}\left(m_{v}+d+1\right)\right] \max _{t_{0}-\tau \leq t \leq t_{0}}\|\varphi(t)-\psi(t)\|^{2}, \quad L_{v}=\max _{-d \leq p_{v} \leq r}\left\{L_{p_{v}}\right\},
$$

Proof. From (2.14) and (2.15) we get

$$
\begin{aligned}
\left\|U_{0}^{(n+1)}\right\|^{2}= & \left\langle U_{0}^{(n)}+\sum_{v=1}^{m} \sum_{i=1}^{s} b_{i}^{[v]} Q_{i}^{[v](n)}, U_{0}^{(n)}+\sum_{v=1}^{m} \sum_{i=1}^{s} b_{i}^{[v]} Q_{i}^{[v](n)}\right\rangle \\
= & \left\|U_{0}^{(n)}\right\|^{2}+2 \sum_{v=1}^{m} \sum_{i=1}^{s} b_{i}^{[v]} \operatorname{Re}\left\langle Q_{i}^{[v](n)}, U_{0}^{(n)}\right\rangle \\
& +\sum_{u, v=1}^{m} \sum_{i, j=1}^{s} b_{i}^{[u]} b_{j}^{[v]}\left\langle Q_{i}^{[u](n)}, Q_{j}^{[v](n)}\right\rangle \\
= & \left\|U_{0}^{(n)}\right\|^{2}+2 \sum_{v=1}^{m} \sum_{i=1}^{s} b_{i}^{[v]} \operatorname{Re}\left\langle Q_{i}^{[v](n)}, U_{i}^{(n)}-\sum_{v=1}^{m} \sum_{j=1}^{s} a_{i j}^{[v]} Q_{j}^{[v](n)}\right\rangle \\
& +\sum_{u, v=1}^{m} \sum_{i, j=1}^{s} b_{i}^{[u]} b_{j}^{[v]}\left\langle Q_{i}^{[v](n)}, Q_{j}^{[u](n)}\right\rangle \\
= & \left\|U_{0}^{(n)}\right\|^{2}+2 \sum_{v=1}^{m} \sum_{i=1}^{s} b_{i}^{[v]} \operatorname{Re}\left\langle Q_{i}^{[v](n)}, U_{i}^{(n)}\right\rangle \\
& -\sum_{u, v=1}^{m} \sum_{i, j=1}^{s}\left(b_{i}^{[u]} a_{i j}^{[v]}+b_{j}^{[v]} a_{i j}^{[u]}-b_{i}^{[u]} b_{j}^{[v]}\right)\left\langle Q_{i}^{[v](n)}, Q_{j}^{[u](n)}\right\rangle .
\end{aligned}
$$

If the matrices $M_{\gamma \mu}$ are nonnegative definite, then

$$
\left\|U_{0}^{(n+1)}\right\|^{2} \leq\left\|U_{0}^{(n)}\right\|^{2}+2 \sum_{v=1}^{m} \sum_{j=1}^{s} b_{j}^{[v]} \operatorname{Re}\left\langle Q_{j}^{[v](n)}, U_{j}^{(n)}\right\rangle .
$$


Furthermore, by conditions (1.2) (1.4) and Schwartz inequality we have

$$
\begin{aligned}
\operatorname{Re}\left\langle Q_{j}^{[v](n)}, U_{j}^{(n)}\right\rangle= & h \operatorname{Re}\left\langle f^{[v]}\left(t_{j}^{(n)}, y_{j}^{(n)}, \tilde{y}_{j}^{[v](n)}, w_{j}^{[v](n)}\right)\right. \\
& \left.-f^{[v]}\left(t_{j}^{(n)}, z_{j}^{(n)}, \tilde{z}_{j}^{[v](n)}, \widehat{w}_{j}^{[v](n)}\right), U_{j}^{(n)}\right\rangle \\
\leq & h \alpha_{v}\left\|U_{j}^{(n)}\right\|^{2}+h \beta_{v}\left\|\tilde{U}_{j}^{[v](n)}\right\|+h \sigma_{v}\left\|w_{j}^{[v](n)}-\widehat{w}_{j}^{[v](n)}\right\|^{2} \\
\leq & h \alpha_{v}\left\|U_{j}^{(n)}\right\|^{2}+h \beta_{v}\left\|\tilde{U}_{j}^{[v](n)}\right\|+2 h^{3} \widetilde{r}_{v}^{2} \eta_{v}^{2} \sigma_{v} \sum_{q=0}^{m_{v}}\left\|U_{j}^{(n-q)}\right\|^{2} \\
= & h \alpha_{v}\left\|U_{j}^{(n)}\right\|^{2}+2 h^{3} \widetilde{r}_{v}^{2} \eta_{v}^{2} \sigma_{v} \sum_{q=0}^{m_{v}}\left\|U_{j}^{(n-q)}\right\|^{2}, \quad t_{n}+c_{j} h-\tau_{v} \leq t_{0} \\
= & h \alpha_{v}\left\|U_{j}^{(n)}\right\|^{2}+h \beta_{v}\left\|\sum_{p_{v}=-d}^{r} L_{p_{v}}\left(\delta_{v}\right) U_{j}^{\left(n-m_{v}+p_{v}\right)}\right\|^{2} \\
& +2 h^{3} \widetilde{r}_{v}^{2} \eta_{v}^{2} \sigma_{v} \sum_{q=0}^{m_{v}}\left\|U_{j}^{(n-q)}\right\|^{2}, t_{n}+c_{j} h-\tau_{v}>t_{0} \\
\leq & h \alpha_{v}\left\|U_{j}^{(n)}\right\|^{2}+2 h^{3} \widetilde{r}_{v}^{2} \eta_{v}^{2} \sigma_{v} \sum_{q=0}^{m_{v}}\left\|U_{j}^{(n-q)}\right\|^{2}, \quad t_{n}+c_{j} h-\tau_{v} \leq t_{0} \\
& +2 h^{3} \widetilde{r}_{v}^{2} \eta_{v}^{2} \sigma_{v} \sum_{q=0}^{m_{v}}\left\|U_{j}^{(n-q)}\right\|^{2}, t_{n}+c_{j} h-\tau_{v}>t_{0} . \\
\leq & h \alpha_{v}\left\|U_{j}^{(n)}\right\|^{2}+2 h \beta_{v} L_{v}^{2}\left\|\sum_{p_{v}=-d}^{r} U_{j}^{\left(n-m_{v}+p_{v}\right)}\right\|^{2} \\
&
\end{aligned}
$$

For (2.23), we have

$$
\begin{aligned}
(2.23) & \leq 2 h \beta_{v} L_{v}^{2}\left\|\sum_{p_{v}=-d}^{r} U_{j}^{\left(n-m_{v}+p_{v}\right)}\right\|^{2}+2 h^{3} \widetilde{r}_{v}^{2} \eta_{v}^{2} \sigma_{v} \sum_{q=0}^{m_{v}}\left\|U_{j}^{(n-q)}\right\|^{2} \\
& \leq 3 h \beta_{v} L_{v}^{2}\left\|\sum_{p_{v}=-d}^{m_{v}} U_{j}^{\left(n-m_{v}+p_{v}\right)}\right\|^{2} .
\end{aligned}
$$

By the same way, we can also get

$$
(2.22) \leq 3 h \beta_{v} L_{v}^{2}\left\|\sum_{P_{v}=-d}^{m_{v}} U_{j}^{\left(n-m_{v}+p_{v}\right)}\right\|^{2} .
$$


Substituting (2.25) and (2.24) into (2.19), yields

$$
\begin{aligned}
\left\|U_{0}^{(n+1)}\right\|^{2} & \leq\left\|U_{0}^{(n)}\right\|^{2}+2 h \sum_{v=1}^{m} \sum_{j=1}^{s} 3 \beta_{v} L_{v}^{2} b_{j}^{[v]}\left\|\sum_{p_{v}=-d}^{m_{v}} U_{j}^{\left(n-m_{v}+p_{v}\right)}\right\|^{2} \\
& \leq\left\|U_{0}^{(n)}\right\|^{2}+6 h \sum_{v=1}^{m} \sum_{j=1}^{s} \beta_{v} L_{v}^{2} b_{j}^{[v]}\left(m_{v}+d+1\right) \max _{-d \leq p_{v} \leq m_{v}}\left\|U_{j}^{\left(n-m_{v}+p_{v}\right)}\right\|^{2} \\
& \leq\left\|U_{0}^{(n)}\right\|^{2}+6 h m s \sum_{v=1}^{m} \beta_{v} L_{v}^{2}\left(m_{v}+d+1\right) \max _{\left(j, p_{v}\right) \in E_{v}}\left\|U_{j}^{\left(n-m_{v}+p_{v}\right)}\right\|^{2} \\
& \leq\left[1+6 h m s \sum_{v=1}^{m} \beta_{v} L_{v}^{2}\left(m_{v}+d+1\right)\right] \max \left\{\left\|U_{0}^{(n)}\right\|^{2}, \max _{\left(j, p_{v}\right) \in E_{v}}\left\|U_{j}^{\left(n-m_{v}+p_{v}\right)}\right\|^{2}\right\},
\end{aligned}
$$

where $E_{v}=\left\{\left(j, P_{v}\right) 1 \leq j \leq s,-d \leq P_{v} \leq r\right\}$.

Similar to (2.27), the inequalities:

$$
\left\|U_{i}^{(n)}\right\|^{2} \leq\left[1+6 h m s \sum_{v=1}^{m} \beta_{v} L_{v}^{2}\left(m_{v}+d+1\right)\right] \max \left\{\left\|U_{0}^{(n)}\right\|^{2}, \max _{\left(j, P_{v}\right) \in E}\left\|U_{j}^{\left(n-m_{v}+P_{v}\right)}\right\|^{2}\right\}
$$

follows for $i=1,2, \ldots, s$.

In the following, with the help of inequalities $(2.27),(2.28)$, and induction we shall prove the inequalities:

$$
\left\|U_{i}^{(n)}\right\|^{2} \leq\left[1+6 h m s \sum_{v=1}^{m} \beta_{v} L_{v}^{2}\left(m_{v}+d+1\right)\right]^{(n+1)} \max _{t_{0}-\tau \leq t \leq t_{0}}\|\varphi(t)-\psi(t)\|^{2},
$$

for $n \geq 0, i=1,2, \ldots, s$.

In fact, it is clear from (2.27), (2.28), and $m_{v} \geq r+1$ such that

$$
\left\|U_{i}^{(0)}\right\|^{2} \leq\left[1+6 h m s \sum_{v=1}^{m} \beta_{v} L_{v}^{2}\left(m_{v}+d+1\right)\right] \max _{t_{0}-\tau \leq t \leq t_{0}}\|\varphi(t)-\psi(t)\|^{2}, \quad i=0,1,2, \ldots, s .
$$

Suppose for $n \leq k(k \geq 0)$ that

$$
\left\|U_{i}^{(n)}\right\|^{2} \leq\left[1+6 h m s \sum_{v=1}^{m} \beta_{v} L_{v}^{2}\left(m_{v}+d+1\right)\right]^{(n+1)} \max _{t_{0}-\tau \leq t \leq t_{0}}\|\varphi(t)-\psi(t)\|^{2}, \quad i=0,1,2, \ldots, s .
$$


Then from (2.27) and (2.28), $m_{v} \geq r+1$ and $1+6 h m s \sum_{v=1}^{m} \beta_{v} L_{v}^{2}\left(m_{v}+d+1\right)>1$, we conclude that

$$
\left\|U_{i}^{(k+1)}\right\|^{2} \leq\left[1+6 h m s \sum_{v=1}^{m} \beta_{v} L_{v}^{2}\left(m_{v}+d+1\right)\right]^{(k+2)} \max _{t_{0}-\tau \leq t \leq t_{0}}\|\varphi(t)-\psi(t)\|^{2}, \quad i=0,1,2, \ldots, s .
$$

This completes the proof of inequalities (2.29). In view of (2.29), we get for $n \geq 0$ that

$$
\begin{aligned}
\left\|U_{0}^{(n)}\right\|^{2} & \leq\left[1+6 h m s \sum_{v=1}^{m} \beta_{v} L_{v}^{2}\left(m_{v}+d+1\right)\right]^{(n+1)} \max _{t_{0}-\tau \leq t \leq t_{0}}\|\varphi(t)-\psi(t)\|^{2} \\
& \leq \exp \left[(n+1) 6 h m s \sum_{v=1}^{m} \beta_{v} L_{v}^{2}\left(m_{v}+d+1\right)\right] \max _{t_{0}-\tau \leq t \leq t_{0}}\|\varphi(t)-\psi(t)\|^{2} \\
& \leq \exp \left[6\left(T-t_{0}\right) m s \sum_{v=1}^{m} \beta_{v} L_{v}^{2}\left(m_{v}+d+1\right)\right] \max _{t_{0}-\tau \leq t \leq t_{0}}\|\varphi(t)-\psi(t)\|^{2} .
\end{aligned}
$$

As a result, we know that method (2.1) is GDN-stable.

\section{D-Convergence}

In order to study the convergence of numerical methods for MDIDEs, we have to mention the concept of the convergence for stiff ODEs.

In 1981, Frank et al. [26] introduced the important concept of B-convergence for numerical methods applied to nonlinear stiff initial value problems of ordinary differential equations. Later, there have been rapid developments in the study of B-convergence, and a significant number of important results have already been found for Runge-Kutta methods. In fact, B-convergence result is nothing but a realistic global error estimate based on onesided Lipschitz constant [27]. In this section, we start discussing the convergence of ARKLM (2.1) for MDIDEs (1.1) with conditions (1.2)-(1.4). The approach to the derivation of these estimates is similar to that used in [25]. We assume the analytic solution $y(t)$ of $(1.1)$ is smooth enough, and its derivatives used later are bounded by

$$
\left\|D^{(i)} y(t)\right\| \leq \widetilde{M}_{i}, \quad t \in\left[t_{0}-\tau, T\right]
$$

where

$$
D^{(i)} y(t)= \begin{cases}y^{(i)}(t), & t \in\left(t_{0}+(j-1) h, t_{0}+j h\right) \\ y^{(i)}\left(t_{0}+j h-0\right), & t=t_{0}+j h\end{cases}
$$


If we introduce some notations

$$
Y^{(n)}=\left[\begin{array}{c}
y\left(t_{n}+c_{1} h\right) \\
y\left(t_{n}+c_{2} h\right) \\
\vdots \\
y\left(t_{n}+c_{s} h\right)
\end{array}\right], \quad \tilde{Y}^{[v](n)}=\left[\begin{array}{c}
y\left(t_{n}+c_{1} h-\tau_{v}\right) \\
y\left(t_{n}+c_{2} h-\tau_{v}\right) \\
\vdots \\
y\left(t_{n}+c_{s} h-\tau_{v}\right)
\end{array}\right], \quad \tilde{w}^{[v](n)}=\left[\begin{array}{c}
w\left(t_{n}+c_{1} h-\tau_{v}\right) \\
w\left(t_{n}+c_{2} h-\tau_{v}\right) \\
\vdots \\
w\left(t_{n}+c_{s} h-\tau_{v}\right)
\end{array}\right] .
$$

With the above notations, the local errors in (2.9) can be defined as

$$
\begin{gathered}
y\left(t_{n+1}\right)=y\left(t_{n}\right)+h \sum_{v=1}^{m} \tilde{b}^{[v]^{T}} f^{[v]}\left(T^{(n)}, Y^{(n)}, \tilde{Y}^{[v](n)}, \tilde{w}^{[v](n)}\right)+Q_{n}, \\
Y^{(n)}=\tilde{e} y\left(t_{n}\right)+h \sum_{v=1}^{m} \tilde{A}^{[v]} f^{[v]}\left(T^{(n)}, Y^{(n)}, \tilde{Y}^{[v](n)}, \tilde{w}^{[v](n)}\right)+r_{n}, \\
\tilde{Y}^{[v](n)}=\left(\tilde{Y}_{1}^{[v](n)}, \tilde{Y}_{2}^{[v](n)}, \ldots, \tilde{Y}_{s}^{[v](n)}\right)^{T},
\end{gathered}
$$

with

$$
\begin{gathered}
\tilde{Y}_{j}^{[v](n)}= \begin{cases}\varphi\left(t_{n}+c_{j} h-\tau_{v}\right), & t_{n}+c_{j} h-\tau_{v} \leq t_{0}, \\
\sum_{P_{v}=-d}^{r} L_{P_{v}}\left(\delta_{v}\right) y_{j}^{\left(n-m_{v}+P_{v}\right)}+\rho_{j}^{[v](n)}, & t_{n}+c_{j} h-\tau_{v}>t_{0},\end{cases} \\
w_{j}^{[v](n)}=h \sum_{q=0}^{m_{v}} d_{q} g^{[v]}\left(t_{n}+c_{j} h, t_{n-q}+c_{j} h, y_{j}^{(n-q)}\right)+R_{j}^{[v](n)} .
\end{gathered}
$$

If we take $\breve{y}_{n}=y\left(t_{n}\right), \breve{y}^{(n)}=Y^{(n)}, \breve{y}^{[v](n)}=\tilde{Y}^{[v](n)}$, and $\breve{w}^{[v](n)}=\widetilde{w}^{[v](n)}$

Then we can get the perturbed scheme of (2.9),

$$
\begin{gathered}
\breve{y}_{n+1}=\breve{y}_{n}+h \sum_{v=1}^{m} \tilde{b}^{[v]^{T}} f^{[v]}\left(T^{(n)}, \breve{y}^{(n)}, \breve{y}^{[v](n)}, \breve{\widetilde{w}}^{[v](n)}\right)+Q_{n}, \\
\breve{y}^{(n)}=\tilde{e} \breve{y}_{n}+h \sum_{v=1}^{m} \tilde{A}^{[v]} f^{[v]}\left(T^{(n)}, \breve{y}^{(n)}, \breve{\breve{y}}^{[v](n)}, \breve{\widetilde{w}}^{[v](n)}\right)+r_{n}, \\
\breve{y}_{j}^{[v](n)}= \begin{cases}\tilde{e} \varphi\left(t_{n}+c_{j} h-\tau_{v}\right), & t_{n}+c_{j} h-\tau_{v} \leq 0, \\
\sum_{P_{v}=-d}^{r} L_{P_{v}}\left(\delta_{v}\right) \breve{y}_{j}^{\left(n-m_{v}+P_{v}\right)}+\rho_{j}^{[v](n)}, & t_{n}+c_{j} h-\tau_{v}>0,\end{cases} \\
w_{j}^{[v](n)}=h \sum_{q=0}^{m_{v}} d_{q} g^{[v]}\left(t_{n}+c_{j} h, t_{n-q}+c_{j} h, y_{j}^{(n-q)}\right)+R_{j}^{[v](n)} .
\end{gathered}
$$


With perturbations, $Q_{n} \in C^{N}, r_{n}=\left(r_{1}^{(n)^{T}}, r_{2}^{(n)^{T}}, \ldots, r_{\mathrm{s}}^{(n)^{T}}\right)^{T}, R^{[v](n)}=\left(R_{1}^{[v](n)}, R_{2}^{[v](n)}, \ldots\right.$, $\left.R_{s}^{[v](n)}\right)^{T}, \rho^{(n)}=\left(\rho_{1}^{(n)^{T}}, \rho_{2}^{(n)^{T}}, \ldots, \rho_{\mathrm{s}}^{(n)^{T}}\right) \in C^{N S}$, according to Taylor formula and the formula in [28, pages 205-212], $Q_{n}, r_{n}$ and $\rho_{n}$ can be determined respectively, as follows:

$$
\begin{gathered}
Q_{n}=\sum_{l=1}^{P} \frac{h^{l}}{(l-1) !}\left(\frac{1}{l}-\sum_{v=1}^{m} \sum_{j=1}^{s} b_{j}^{[v]} c_{j}^{l-1}\right) D^{(l)} y\left(t_{n}\right)+R_{0}^{(n)}, \\
r_{i}^{(n)}=\sum_{l=1}^{P} \frac{h^{l}}{(l-1) !}\left(\frac{1}{l} c_{i}^{l}-\sum_{v=1}^{m} \sum_{j=1}^{s} a_{i j}^{[v]} c_{j}^{l-1}\right) D^{(l)} y\left(t_{n}\right)+R_{i}^{(n)}, \\
\rho_{i}^{(n)}=\frac{h^{q+1}}{(q+1) !} \sum_{v=1}^{m} \prod_{P_{v}=-d}^{r}\left(\delta_{v}-P_{v}\right) D^{(q+1)} y\left(\xi_{i}^{(n)}\right), \quad \xi_{i}^{(n)} \in\left(t_{n-m_{v}-d}+c_{i} h, t_{n-m_{v}+r}+c_{i} h\right),
\end{gathered}
$$

where $q=d+r, R_{i}^{(n)}$, and $\xi_{i}^{(n)}$ satisfy $\left\|R_{i}^{(n)}\right\| \leq \widehat{M}_{i} h^{i+1}, i=0,1,2, \ldots, s, h \in\left(0, h_{0}\right], h_{0}$ depends only on the method, and $\widetilde{M}_{i}(i=0,1,2, \ldots, s)$ depends only on the method and some $\widetilde{M}_{i}$ in (3.2).

Combining (2.2), (2.3), (2.5), and (2.6) with (3.9), (3.10), (3.11), and (3.12) yields the following recursion scheme for the $\varepsilon_{0}^{(n+1)}=\breve{y}_{n+1}-y_{n+1}$ :

$$
\begin{gathered}
\varepsilon_{0}^{(n+1)}=\varepsilon_{0}^{(n)}+h \sum_{v=1}^{m} \widetilde{b}^{[v]^{T}}\left\{f^{[v]}\left(T^{(n)}, \breve{y}^{(n)}, \breve{\breve{y}}^{[v](n)}, w^{[v](n)}\right)-f^{[v]}\left(T^{(n)}, \breve{y}^{(n)}, \tilde{y}^{[v](n)}, w^{[v](n)}\right)\right. \\
\left.+g_{n}^{[v]} \varepsilon_{n}+H^{[v](n)}\left(\breve{w}^{[v](n)}-w^{[v](n)}\right)\right\}+Q n, \\
\varepsilon_{n}=\tilde{e} \varepsilon_{0}^{(n)}+h \sum_{v=1}^{m} \tilde{A}^{[v]}\left\{f^{[v]}\left(T^{(n)}, \breve{y}^{(n)}, \breve{y}^{[v](n)}, w^{[v](n)}\right)-f^{[v]}\left(T^{(n)}, \breve{y}^{(n)}, \tilde{y}^{[v](n)}, w^{[v](n)}\right)\right. \\
\left.+g_{n}^{[v]} \varepsilon_{n}+H^{[v](n)}\left(\breve{\widetilde{w}}^{[v](n)}-w^{[v](n)}\right)\right\}+r_{n},
\end{gathered}
$$

where $\varepsilon_{0}^{(n+1)}=\breve{y}_{n+1}-y_{n+1}, \varepsilon_{n}=\left(\varepsilon_{1}^{(n)^{T}}, \varepsilon_{2}^{(n)^{T}}, \ldots, \varepsilon_{S}^{(n)^{T}}\right)^{T}=\breve{y}^{(n)}-y^{(n)}$

$$
\begin{gathered}
g_{i}^{[v](n)}=\int_{0}^{1} f_{2}\left(t_{n}+c_{i} h, y_{i}^{(n)}+\theta\left(\breve{y}_{i}^{(n)}-y_{i}^{(n)}\right), \breve{\breve{y}}_{i}^{[v](n)}, \breve{\widetilde{w}}_{i}^{[v](n)}\right) d \theta, \quad i=1,2, \ldots, s, \\
H_{i}^{[v](n)}=\int_{0}^{1} f_{4}\left(t_{n}+c_{i} h, y_{i}^{(n)}, \breve{y}_{i}^{[v](n)}, \breve{\widetilde{w}}_{i}^{[v](n)}+\theta\left(\breve{\widetilde{w}}_{i}^{[v](n)}-w_{i}^{[v](n)}\right)\right) d \theta,
\end{gathered}
$$


here, $f_{i}\left(x_{1}, x_{2}, x_{3}, x_{4}\right)$ is the Jacobian matrix $\left(\partial f\left(x_{1}, x_{2}, x_{3}, x_{4}\right) / \partial x_{i}\right) i=1,2,3,4$.

$$
\begin{aligned}
H^{[v](n)}\left(\breve{\widetilde{w}}^{[v](n)}-w^{[v](n)}\right)= & h H^{[v](n)} \sum_{q=1}^{m_{v}} d_{q}\left[g^{[v]}\left(t_{n}, t_{n-q}, \breve{y}^{(n-q)}\right)-g^{[v]}\left(t_{n}, t_{n-q}, y^{(n-q)}\right)\right] \\
& +h H^{[v](n)} R^{[v](n)} \\
& +h H^{[v](n)} d_{0}\left[g^{[v]}\left(t_{n}, t_{n}, \breve{y}^{(n)}\right)-g^{[v]}\left(t_{n}, t_{n}, y^{(n)}\right)\right] \\
= & h H^{[v](n)} \sum_{q=1}^{m_{v}} d_{q}\left[g^{[v]}\left(t_{n}, t_{n-q}, \breve{y}^{(n-q)}\right)-g^{[v]}\left(t_{n}, t_{n-q}, y^{(n-q)}\right)\right] \\
& +h H^{[v](n)} R^{[v](n)} \\
& +h H^{[v](n)} d_{0} \int_{0}^{1} g_{3}^{[v]}\left(t_{n}, t_{n}, y^{(n)}+\theta\left(\breve{y}^{(n)}-y^{(n)}\right)\right) d \theta \cdot \varepsilon_{n} .
\end{aligned}
$$

Assume that $\left(\tilde{I}_{s}-h \sum_{v=1}^{m} \tilde{A}^{[v]}\left(g^{[v](n)}+h H^{[v](n)} d_{0} g_{3}^{[v](n)}\right)\right)$ is regular, from (3.16) and (3.17), (3.18), we can get

$$
\begin{aligned}
\varepsilon_{0}^{(n+1)}= & \left\{I_{N}+h \sum_{v=1}^{m} \tilde{b}^{[v]^{T}}\left[\tilde{I}_{s}-h \sum_{v=1}^{m} \tilde{A}^{[v]}\left(g^{[v](n)}+h H^{[v](n)} d_{0} g_{3}^{[v](n)}\right)\right]^{-1}\right. \\
& \left.\times \tilde{e}\left(g^{[v](n)}+h H^{[v](n)} d_{0} g_{3}^{[v](n)}\right)\right\} \varepsilon_{0}^{(n)} \\
& +h \sum_{v=1}^{m} \tilde{b}^{[v]^{T}}\left(g^{[v](n)}+h H^{[v](n)} d_{0} g_{3}^{[v](n)}\right)\left[\tilde{I}_{s}-h \sum_{v=1}^{m} \tilde{A}^{[v]}\left(g^{[v](n)}+h H^{[v](n)} d_{0} g_{3}^{[v](n)}\right)\right]^{-1} \\
& \times\left(r_{n}+h^{2} \sum_{v=1}^{m} \tilde{A}^{[v]} H^{[v](n)} R^{[v](n)}\right) \\
& +h \sum_{v=1}^{m} \tilde{b}^{[v]^{T}}\left[\tilde{I}_{s}-h \sum_{v=1}^{m} \tilde{A}^{[v]}\left(g^{[v](n)}+h H^{[v](n)} d_{0} g_{3}^{[v](n)}\right)\right]^{-1} \\
& \left.\times h \sum_{v=1}^{m} \tilde{A}^{[v]}\left(g^{[v](n)}+h H^{[v](n)} d_{0} g_{3}^{[v](n)}\right)\right] \\
& +\left\{f^{[v]}\left(T^{(n)}, \breve{y}^{(n)}, \tilde{y}^{[v](n)}, w^{[v](n)}\right)-f^{[v]}\left(T^{(n)}, \breve{y}^{(n)}, \tilde{y}^{[v](n)}, w^{[v](n)}\right)\right. \\
& \left.+h H^{[v](n)} \sum_{q=1}^{m_{v}} d_{q}\left[g^{[v]}\left(t_{n}, t_{n-q}, \breve{y}^{(n-q)}\right)-g^{[v]}\left(t_{n}, t_{n-q}, y^{(n-q)}\right)\right]\right\} \\
+ & Q_{n}+h^{2} \sum_{v=1}^{m} \tilde{b}^{[v]^{T}} H^{[v](n)} R^{[v](n)} .
\end{aligned}
$$

Now, we introduce the concept of D-convergence from [25]. 
Definition 3.1. An ARKLM (2.1) with $y_{n}=y\left(t_{n}\right)(n \leq 0), y_{i}^{(n)}=y\left(t_{n}+c_{i} h\right)(n<0)$ and $\tilde{y}_{i}^{[v](n)}=y\left(t_{n}+c_{i} h-\tau_{v}\right)(n<0)$ is called D-convergence of order $p$ if this method, when applied to any given DIDEs (1.1) subject to (1.2)-(1.4); produce an approximation sequence $y_{n}$ and the global error satisfies a bound of the form:

$$
\left\|y\left(t_{n}\right)-y_{n}\right\| \leq C\left(t_{n}\right) h^{P}, \quad h \in\left(0, h_{0}\right]
$$

where the maximum stepsize $h_{0}$ depends on characteristic parameter $\alpha_{v}, \beta_{v}, \sigma_{v}, r_{v}, \tilde{r}_{v}$ and the method, the function $C(t)$ depends only on some $\widetilde{M}_{i}$ in (3.2), delay $\tau_{v}$, characteristic parameters $\alpha_{v}, \beta_{v}, \sigma_{v}, r_{v}, \tilde{r}_{v}, v=1,2, \ldots, m$, and the method.

Definition 3.2. The ARKLM (2.2), (2.3), (2.5), and (2.6) is said to be DA-stable if the matrix $\left(I_{s}-\sum_{v=1}^{m} A^{[v]} \xi\right)$ is regular for $\xi \in C^{-}:=\{\xi \in C \mid \operatorname{Re} \xi \leq 0\}$, and $\left|R_{i}(\xi)\right| \leq 1$ for all $\xi \in$ $C^{-}, i=0,1, \ldots, s$.

Where

$$
\begin{gathered}
R_{i}\left(\varepsilon_{1}\right)=1+\sum_{v=1}^{m} A_{i}^{[v]} \varepsilon_{1}\left(I_{S}-\sum_{v=1}^{m} A^{[v]} \xi\right)^{-1} e \\
A_{0}^{[v]}=b^{[v]}, \quad A_{i}^{[v]}=\left(a_{i 1}^{[v]}, a_{i 2}^{[v]}, \ldots, a_{i s}^{[v]}\right)^{T}, \quad i=0,1, \ldots, s .
\end{gathered}
$$

Definition 3.3. The ARKLM (2.2), (2.3), (2.5), and (2.6) is said to be ASI-stable if the matrix $\left(I_{s}-\sum_{v=1}^{M} A^{[v]} \xi\right)$ is regular for $\xi \in C^{-}$, and $\left(I_{s}-\sum_{v=1}^{M} A^{[v]} \xi\right)^{-1}$ is uniformly bounded for $\xi \in C^{-}$.

Definition 3.4. The ARKLM (2.2), (2.3), (2.5), and (2.6) is said to be DAS-stable if the $\operatorname{matrix}\left(I_{s}-\sum_{v=1}^{M} A^{[v]} \xi\right)$ is regular for $\xi \in C^{-}$, and $\sum_{v=1}^{m} A_{i}^{[\nu]^{T}} \xi\left(I_{s}-\sum_{v=1}^{M} A^{[v]} \xi\right)^{-1}(i=0,1, \ldots, \mathrm{s})$ is uniformly bounded for $\xi \in C^{-}$.

Lemma 3.5. Suppose the ARKLM (2.2), (2.3), (2.5), and (2.6) is DA-DAS- and ASI-stable, then there exist positive constants $h_{0}, \gamma_{1}, \gamma_{2}, \gamma_{3}$, which depend only on the method and the parameter $\alpha_{v}, \beta_{v}, \sigma_{v}, r_{v}, \tilde{r}_{v}$ such that

$$
\begin{gathered}
\left\|\tilde{I}_{s}-\sum_{v=1}^{M} \tilde{A}^{[v]} \xi\right\| \leq \gamma_{1}, \\
\left\|I_{N}+\sum_{v=1}^{m} \tilde{A}_{i}^{[v]^{T}} \xi\left(\tilde{I}_{s}-\sum_{v=1}^{m} \tilde{A}^{[v]} \xi\right)^{-1} \tilde{e}\right\| \leq 1+\gamma_{2} h, \\
\left\|\sum_{v=1}^{m} \tilde{A}_{i}^{[v]^{T}} \xi\left(\tilde{I}_{S}-\sum_{v=1}^{m} \tilde{A}^{[v]} \xi\right)^{-1} v\right\| \leq \gamma_{3}\|v\|, \quad v \in C^{N S}, \\
h \in\left(0, h_{0}\right], \quad i=0,1,2 \ldots, s .
\end{gathered}
$$

Proof. This Lemma can be proved in the similar way as that of in [29, Lemmas 3.5-3.7]. 
Theorem 3.6. Suppose the ARKLM (2.2), (2.3), (2.5), and (2.6) is DA-DAS- and ASI-stable, then there exist positive constants $h_{0}, \gamma_{3}, \gamma_{4}, \gamma_{5}$, which depend only on the method and the parameters $\alpha_{v}, \beta_{v}, \sigma_{v}, r_{v}, \tilde{r}_{v}$, such that for $h \in\left(o, h_{0}\right]$,

$$
\left\|\varepsilon_{i}^{(n)}\right\| \leq\left\{\begin{array}{c}
\left(1+h \gamma_{4}\right) \max \left\{\left\|\varepsilon_{0}^{(n+1)}\right\|, \max _{\left(i, p_{v}\right) \in E}\left\|\varepsilon_{i}^{\left(n-m_{v}+p_{v}\right)}\right\|, \max _{(i, q) \in E_{q}}\left\|\varepsilon_{i}^{(n-q)}\right\|\right\}+h \gamma_{5} \max _{1 \leq i \leq s}\left\|\rho_{i}^{(n-1)}\right\| \\
+\left\|\widetilde{Q}_{n-1}\right\|+\gamma_{3}\left\|\widetilde{\gamma}_{n-1}\right\|, \quad i=0, \\
\left(1+h \gamma_{4}\right) \max \left\{\left\|\varepsilon_{0}^{(n+1)}\right\|, \max _{\left(i, p_{v}\right) \in E}\left\|\varepsilon_{i}^{\left(n-m_{v}+p_{v}\right)}\right\|, \max _{(i, q) \in E_{q}}\left\|\varepsilon_{i}^{(n-q)}\right\|\right\}+h \gamma_{5} \max _{1 \leq i \leq s}\left\|\rho_{i}^{(n)}\right\| \\
+\left\|\widetilde{Q}_{n}\right\|+\gamma_{3}\left\|\tilde{\gamma}_{n}\right\|, \quad i=1,2, \ldots, s,
\end{array}\right.
$$

where $\varepsilon_{0}^{(n)}=\breve{y}_{n}-y_{n}, \varepsilon_{i}^{(n)}=\breve{y}_{i}^{(n)}-y_{i}^{(n)}, E=\left\{\left(i, p_{v}\right) \mid 1 \leq i \leq s,-d \leq p_{v} \leq \gamma\right\}, E_{q}=\{(i, q) \mid 1 \leq$ $i \leq s, 1 \leq q \leq m\}, \widetilde{Q}_{n}=Q_{n}+h^{2} \sum_{v=1}^{m} \tilde{b}^{[v]^{T}} H^{[v](n)} R^{[v](n)}, \widetilde{r}_{n}=r_{n}+h^{2} \sum_{v=1}^{m} \widetilde{A}^{[v]} H^{[v](n)} R^{[v](n)}$.

Proof. Using (3.19) and Lemma 3.5, for $h \in\left(0, h_{0}\right]$, we obtain that

$$
\begin{aligned}
& \varepsilon_{0}^{(n+1)} \leq\left(1+\gamma_{2} h\right)\left\|\varepsilon_{0}^{(n)}\right\|+\gamma_{3}\left\|\tilde{r}_{n}\right\|+\left\|\widetilde{Q}_{n}\right\| \\
& +h \gamma_{3} \| \sum_{v=1}^{m} \tilde{A}^{[v]}\left\{f^{[v]}\left(T^{(n)}, \breve{y}^{(n)}, \breve{y}^{[v](n)}, w^{[v](n)}\right)-f^{[v]}\left(T^{(n)}, \breve{y}^{(n)}, \tilde{y}^{[v](n)}, w^{[v](n)}\right)\right. \\
& \left.+h H^{[v](n)} \sum_{q=1}^{m_{v}} d_{q}\left[g^{[v]}\left(t_{n}, t_{n-q}, \breve{y}^{(n-q)}\right)-g^{[v]}\left(t_{n}, t_{n-q}, y^{(n-q)}\right)\right]\right\} \| \\
& +h \| \sum_{v=1}^{m} \tilde{b}^{[v]^{T}}\left\{f^{[v]}\left(T^{(n)}, \breve{y}^{(n)}, \breve{y}^{[v](n)}, w^{[v](n)}\right)-f^{[v]}\left(T^{(n)}, \breve{y}^{(n)}, \tilde{y}^{[v](n)}, w^{[v](n)}\right)\right. \\
& \left.+h H^{[v](n)} \cdot \sum_{q=1}^{m_{v}} d_{q}\left[g^{[v]}\left(t_{n}, t_{n-q}, \breve{y}^{(n-q)}\right)-g^{[v]}\left(t_{n}, t_{n-q}, y^{(n-q)}\right)\right]\right\} \| \\
& \leq\left(1+\gamma_{2} h\right)\left\|\varepsilon_{0}^{(n)}\right\|+\gamma_{3}\left\|\tilde{r}_{n}\right\|+\left\|\widetilde{Q}_{n}\right\| \\
& +h \gamma_{3} \sum_{v=1}^{m}\left\{\sum_{i=1}^{s} \| \sum_{j=1}^{s} a_{i j}^{[v]}\left[f^{[v]}\left(t_{j}^{(n)}, y_{j}^{(n)}, \breve{y}_{j}^{[v](n)}, w_{j}^{[v](n)}\right)-f^{[v]}\left(t_{j}^{(n)}, y_{j}^{(n)}, \tilde{y}_{j}^{[v](n)}, w_{j}^{[v](n)}\right)\right]\right. \\
& \left.+h H_{j}^{[v](n)} \sum_{q=1}^{m_{V}} d_{q}\left[g^{[v]}\left(t_{n}, t_{n-q}, \breve{y}_{j}^{(n-q)}\right)-g^{[v]}\left(t_{n}, t_{n-q}, y_{j}^{(n-q)}\right)\right] \|^{2}\right\}^{1 / 2}
\end{aligned}
$$




$$
\begin{aligned}
+h \sum_{v=1}^{m} \| \sum_{j=1}^{s} b_{j}^{[v]}\left\{f^{[v]}\left(t_{j}^{(n)}, y_{j}^{(n)}, \breve{\breve{y}}_{j}^{[v](n)}, w_{j}^{[v](n)}\right)-f^{[v]}\left(t_{j}^{(n)}, y_{j}^{(n)}, \tilde{y}_{j}^{[v](n)}, w_{j}^{[v](n)}\right)\right. \\
\left.+h H_{j}^{[v](n)} \sum_{q=1}^{m_{V}} d_{q}\left[g^{[v]}\left(t_{n}, t_{n-q}, \breve{y}_{j}^{(n-q)}\right)-g^{[v]}\left(t_{n}, t_{n-q}, y_{j}^{(n-q)}\right)\right]\right\} \| .
\end{aligned}
$$

For

$$
\begin{aligned}
h \gamma_{3} \sum_{v=1}^{m}\left\{\sum_{i=1}^{s} \| \sum_{j=1}^{s} a_{i j}^{[v]}\left[f^{[v]}\left(t_{j}^{(n)}, y_{j}^{(n)}, \breve{y}_{j}^{[v](n)}, w_{j}^{[v](n)}\right)-f^{[v]}\left(t_{j}^{(n)}, y_{j}^{(n)}, \tilde{y}_{j}^{[v](n)}, w_{j}^{[v](n)}\right)\right]\right. \\
\left.+h H_{j}^{[v](n)} \sum_{q=1}^{m_{V}} d_{q}\left[g^{[v]}\left(t_{n}, t_{n-q}, \breve{y}_{j}^{(n-q)}\right)-g^{[v]}\left(t_{n}, t_{n-q}, y_{j}^{(n-q)}\right)\right] \|^{2}\right\}^{1 / 2}=(3.25 \mathrm{~b}) .
\end{aligned}
$$

Then

$$
\begin{aligned}
& (3.25 \mathrm{~b}) \leq h \gamma_{3} \sum_{v=1}^{m}\left\{\sum _ { i = 1 } ^ { s } 2 \sum _ { j = 1 } ^ { s } | a _ { i j } ^ { [ v ] } | ^ { 2 } \left\{\| f^{[v]}\left(t_{j}^{(n)}, y_{j}^{(n)}, \breve{\breve{y}}_{j}^{[v](n)}, w_{j}^{[v](n)}\right)\right.\right. \\
& -f^{[v]}\left(t_{j}^{(n)}, y_{j}^{(n)}, \tilde{y}_{j}^{[v](n)}, w_{j}^{[v](n)}\right) \|^{2} \\
& +\| h H_{j}^{[v](n)} \sum_{q=1}^{m_{V}} d_{q}\left[g^{[v]}\left(t_{n}, t_{n-q}, \breve{y}_{j}^{(n-q)}\right)\right. \\
& \left.\left.\left.-g^{[v]}\left(t_{n}, t_{n-q}, y_{j}^{(n-q)}\right)\right]\|\|^{2}\right\}\right\}^{1 / 2} \\
& \leq h \gamma_{3} \sum_{v=1}^{m}\left\{\sum _ { i = 1 } ^ { s } \left\{2 \sum_{j=1}^{s}\left|a_{i j}^{[v]}\right|^{2} r_{v}^{2}\left\|\breve{y}_{j}^{[v](n)}-\tilde{y}_{j}^{[v](n)}\right\|^{2}+2 \sum_{j=1}^{s}\left|a_{i j}^{[v]}\right|^{2} h^{2} H_{j}^{[v](n)^{2}} 2 \sum_{q=1}^{m_{v}} d_{q}\right.\right. \\
& \left.\left.\cdot\left\|g^{[v]}\left(t_{n}, t_{n-q}, \breve{y}_{j}^{(n-q)}\right)-g^{[v]}\left(t_{n}, t_{n-q}, y_{j}^{(n-q)}\right)\right\|^{2}\right\}\right\}^{1 / 2} \\
& \leq h \gamma_{3} \sum_{v=1}^{m} \sqrt{\sum_{i=1}^{s} 2} \sum_{j=1}^{s}\left|a_{i j}^{[v]}\right|^{2} r_{v}^{2}\left\|\breve{y}_{j}^{[v](n)}-\tilde{y}_{j}^{[v](n)}\right\|^{2} \\
& +2 h^{2} \gamma_{3} \sum_{v=1}^{m} \sqrt{\sum_{i=1}^{s} \sum_{j=1}^{s}\left|a_{i j}^{[v]}\right|^{2} H_{j}^{[v](n)^{2}} \sum_{q=1}^{m_{v}} d_{q}^{2} \cdot\left\|g^{[v]}\left(t_{n}, t_{n-q}, \breve{y}_{j}^{(n-q)}\right)-g^{[v]}\left(t_{n}, t_{n-q}, y_{j}^{(n-q)}\right)\right\|^{2}}
\end{aligned}
$$




$$
\begin{aligned}
\leq & 2 h \gamma_{3} \sum_{v=1}^{m} \sum_{i=1}^{s} \sum_{j=1}^{s}\left|a_{i j}^{[v]}\right| r_{v}\left\|\breve{y}_{j}^{[v](n)}-\tilde{y}_{j}^{[v](n)}\right\| \\
& +2 h^{2} \gamma_{3} \sum_{v=1}^{m} \sum_{i=1}^{s} \sum_{j=1}^{s} \sum_{q=1}^{m_{v}} d_{q}\left|a_{i j}^{[v]}\right|\left|H_{j}^{[v](n)}\right| \cdot\left\|g^{[v]}\left(t_{n}, t_{n-q}, \breve{y}_{j}^{(n-q)}\right)-g^{[v]}\left(t_{n}, t_{n-q}, y_{j}^{(n-q)}\right)\right\| \\
\leq & 2 h \gamma_{3} \sum_{v=1}^{m} \sum_{i, j=1}^{s}\left|a_{i j}^{[v]}\right| r_{v}\left\|\breve{\widetilde{y}}_{j}^{[v](n)}-\tilde{y}_{j}^{[v](n)}\right\| \\
& +2 h^{2} \gamma_{3} \sum_{q=1}^{m_{v}} d_{q} \sum_{v=1}^{m} \sum_{i, j=1}^{s}\left|a_{i j}^{[v]}\right|\left|H_{j}^{[v](n)}\right| \widetilde{r}_{v}\left\|\breve{y}_{j}^{(n-q)}-y_{j}^{(n-q)}\right\| .
\end{aligned}
$$

For

$$
\begin{array}{r}
h \sum_{v=1}^{m} \| \sum_{j=1}^{s} b_{j}^{[v]}\left\{f^{[v]}\left(t_{j}^{(n)}, y_{j}^{(n)}, \breve{\breve{y}}_{j}^{[v](n)}, w_{j}^{[v](n)}\right)-f^{[v]}\left(t_{j}^{(n)}, y_{j}^{(n)}, \tilde{y}_{j}^{[v](n)}, w w_{j}^{[v](n)}\right)\right. \\
+h H_{j}^{[v](n)} \sum_{q=1}^{m_{V}} d_{q}\left[g^{[v]}\left(t_{n}, t_{n-q}, \breve{y}_{j}^{(n-q)}\right)\right. \\
\left.\left.-g^{[v]}\left(t_{n}, t_{n-q}, y_{j}^{(n-q)}\right)\right]\right\} \|=(3.25 c),
\end{array}
$$

then

$$
\begin{aligned}
(3.25 \mathrm{c}) \leq & h \sum_{v=1}^{m} \sum_{j=1}^{s}\left|b_{j}^{(v)}\right| \gamma_{v}\left\|\breve{y}_{j}^{[v](n)}-\tilde{y}_{j}^{[v](n)}\right\| \\
& +h^{2} \sum_{v=1}^{m} \sum_{j=1}^{s}\left|b_{j}^{[v]}\right|\left|H_{j}^{[v](n)}\right|\left\{\sum_{q=1}^{m_{v}} d_{q}\left\|g^{[v]}\left(t_{n}, t_{n-q}, \breve{y}_{j}^{(n-q)}\right)-g^{[v]}\left(t_{n}, t_{n-q}, y_{j}^{(n-q)}\right)\right\|\right\} \\
\leq & h \sum_{v=1}^{m} \sum_{j=1}^{s}\left|b_{j}^{(v)}\right| \gamma_{v}\left\|\breve{y}_{j}^{[v](n)}-\tilde{y}_{j}^{[v](n)}\right\|+h^{2} \sum_{v=1}^{m} \sum_{j=1}^{s}\left|b_{j}^{[v]}\right|\left|H_{j}^{[v](n)}\right|\left\{\sum_{q=1}^{m_{v}} d_{q} \tilde{r}_{v}\left\|\breve{y}_{j}^{(n-q)}-y_{j}^{(n-q)}\right\|\right\} .
\end{aligned}
$$

Combine (3.27), (3.29), and (3.25a), we have

$$
\begin{aligned}
\varepsilon_{0}^{(n+1)} \leq & \left(1+\gamma_{2} h\right)\left\|\varepsilon_{0}^{(n)}\right\|+\gamma_{3}\left\|\tilde{r}_{n}\right\|+\left\|\tilde{Q}_{n}\right\| \\
& +h \sum_{v=1}^{m} \gamma_{v}\left(2 \gamma_{3} \sum_{i, j=1}^{s}\left|a_{i j}^{[v]}\right|+\sum_{j=1}^{s}\left|b_{j}^{[v]}\right|\right)\left\|\widetilde{y}_{j}^{[v](n)}-\tilde{y}_{j}^{[v](n)}\right\| \\
& +h^{2} \sum_{v=1}^{m} \tilde{r}_{v} \sum_{q=1}^{m_{v}} d_{q} \sum_{j=1}^{s}\left\{2 \gamma_{3} \sum_{i=1}^{s}\left|a_{i j}^{[v]}\right| \cdot\left|H_{j}^{[v](n)}\right|+\left|b_{j}^{[v]}\right| \cdot\left|H_{j}^{[v](n)}\right|\right\}\left\|\varepsilon_{j}^{(n-q)}\right\| .
\end{aligned}
$$


Moreover, it follows from (2.5) and (3.11) that

$$
\left\|\breve{y}_{j}^{[v](n)}-\widetilde{y}_{j}^{[v](n)}\right\| \leq \sup _{\delta_{v} \in[0,1)} \sum_{P_{v}=-d}^{r}\left|L_{p_{v}}\left(\delta_{v}\right)\right| \max _{-d \leq P_{r} \leq r}\left\|\varepsilon_{j}^{\left(n-m_{v}+P_{v}\right)}\right\|+\left\|\rho_{j}^{[v](n)}\right\| .
$$

Substituting (3.31) in (3.30), we get

$$
\begin{aligned}
\left\|\varepsilon_{0}^{(n+1)}\right\| & \leq\left(1+\gamma_{4}^{(0)} h+\gamma_{6}^{(0)} h^{2}\right) \max \left\{\left\|\varepsilon_{0}^{(n)}\right\|, \max _{\left(j, p_{v}\right) \in E}\left\|\varepsilon_{j}^{\left(n-m_{v}+P_{v}\right)}\right\|, \max _{(j . q) \in E_{q}}\left\|\varepsilon_{j}^{(n-q)}\right\|\right\}+\left\|\widetilde{Q}_{n}\right\| \\
& +\gamma_{3}\left\|\widetilde{r}_{n}\right\|+h \gamma_{5}^{(0)} \max _{(j, v) \in E_{m}}\left\|\rho_{j}^{[v](n)}\right\|, \quad h \in\left(0, h_{0}\right],
\end{aligned}
$$

where

$$
\begin{gathered}
\gamma_{4}^{(0)}=\gamma_{2}+\gamma_{5}^{(0)} \sup _{\delta_{v \in[0,1)}} \sum_{P_{v}=-d}^{r}\left|L_{p_{v}}\left(\delta_{v}\right)\right|, \quad \gamma_{5}^{(0)}=\sum_{v=1}^{m} r_{v}\left(2 \gamma_{3} \sum_{i, j=1}^{s}\left|a_{i j}^{[v]}\right|+\sum_{j=1}^{s}\left|b_{j}^{[v]}\right|\right), \\
\gamma_{6}^{(0)}=\sum_{v=1}^{m} \tilde{\gamma}_{v} \sum_{q=1}^{m_{v}} \sum_{j=1}^{s} d_{q}\left\{2 \gamma_{3} \sum_{i=1}^{s}\left|a_{i j}[v] \cdot H_{j}^{[v](n)}\right|+\left|b_{j}^{[v]} \cdot H_{j}^{[v](n)}\right|\right\}, \\
E=\left\{\left(j, P_{v}\right) \mid 1 \leq j \leq s,-d \leq P_{v} \leq r\right\}, \quad E_{q}=\left\{(j, q) \mid 1 \leq j \leq s, 1 \leq q \leq m_{v}\right\}, \\
E_{m}=\{(j, v) \mid 1 \leq j \leq s, 1 \leq v \leq m\} .
\end{gathered}
$$

By Lemma 3.5, similar to (3.32), we can obtain the inequalities:

$$
\begin{aligned}
\left\|\varepsilon_{i}^{(n)}\right\| \leq & \left(1+h \gamma_{4}^{(i)}+h^{2} \gamma_{6}^{(i)}\right) \max \left\{\left\|\varepsilon_{0}^{(n)}\right\|, \max _{\left(j, P_{v} \in E\right)}\left\|\varepsilon_{j}^{\left(n-m_{v}+P_{v}\right)}\right\|, \max _{(j, q) \in E_{q}}\left\|\varepsilon_{j}^{(n-q)}\right\|\right\} \\
& +h \gamma_{5}^{(i)} \max _{(j, v) \in E_{m}}\left\|\rho_{j}^{[v](n)}\right\|+\left\|\widetilde{Q}_{n}\right\|+\gamma_{3}\left\|\widetilde{r}_{n}\right\|, \quad i=1,2, \ldots, s, h \in\left(0, h_{0}\right]
\end{aligned}
$$

where

$$
\begin{gathered}
\gamma_{4}^{(i)}=\gamma_{2}+\gamma_{5}^{(i)} \sup _{\delta_{v \in[0,1)}} \sum_{P_{v}=-d}^{r}\left|L_{p_{v}}\left(\delta_{v}\right)\right|, \quad \gamma_{5}^{(i)}=\sum_{v=1}^{m} r_{v}\left(2 \gamma_{3} \sum_{i, j=1}^{s}\left|a_{i j}^{[v]}\right|+\sum_{j=1}^{s}\left|a_{i j}^{[v]}\right|\right), \\
\gamma_{6}^{(i)}=\sum_{v=1}^{m} \tilde{\gamma}_{v} \sum_{q=1}^{m_{v}} \sum_{j=1}^{s} d_{q}\left(2 \gamma_{3} \sum_{i=1}^{s}\left|a_{i j}{ }^{[v]} \cdot H_{j}^{[v](n)}\right|+\left|a_{i j}^{[v]} \cdot H_{j}^{[v](n)}\right|\right) .
\end{gathered}
$$

Setting $\gamma_{4}=\max _{0 \leq i \leq s} \gamma_{4}^{(i)}, \gamma_{5}=\max _{0 \leq i \leq s} \gamma_{5}^{(i)}, \gamma_{6}=\max _{0 \leq i \leq s} \gamma_{6}^{(i)}$.

Combining (3.32) with (3.34), we immediately obtain the conclusion of this theorem. 
Now, we turn to study the convergence of ARKLM (2.1) for (1.1). It is always assumed that the analytic solution $y(t)$ of (1.1) is smooth enough on each internal of the form $\left(t_{0}+(j-\right.$ $\left.1) h, t_{0}+j h\right)$ ( $j$ is a positive integer) as (3.2) defined.

Theorem 3.7. Assume ARKLM (2.1) with stage order $p$ is DA-, DAS- and ASI-stable, then the $A R K L M(2.1)$ is D-convergent of order $\min \{p, q+1, s+1\}$, where $q=d+r$.

Proof. By Theorem 3.6, we have for $h \in\left(0, h_{0}\right]$

$$
\left\|\varepsilon_{i}^{(n)}\right\| \leq\left\{\begin{array}{c}
\left(1+h \gamma_{4}+h^{2} \gamma_{6}\right) \max \left\{\left\|\varepsilon_{0}^{(n-1)}\right\|, \max _{\left(i, p_{v}\right) \in E}\left\|\varepsilon_{i}^{\left(n-1-m_{v}+p_{v}\right)}\right\|, \max _{\left(j, q \in E_{q}\right)}\left\|\varepsilon_{j}^{(n-q)}\right\|\right\} \\
+T_{1} h^{p+1}+T_{2} h^{q+2}+T_{3} h^{s+2}, \quad i=0 \\
\left(1+h \gamma_{4}+h^{2} \gamma_{6}\right) \max \left\{\left\|\varepsilon_{0}^{(n)}\right\|, \max _{\left(i, p_{v}\right) \in E}\left\|\varepsilon_{i}^{\left(n-m_{v}+p_{v}\right)}\right\|, \max _{(j, q) \in E_{q}}\left\|\varepsilon_{j}^{(n-q)}\right\|\right\} \\
+T_{1} h^{p+1}+T_{2} h^{q+2}+T_{3} h^{s+2}, \quad i=1,2, \ldots, s,
\end{array}\right.
$$

where

$$
\begin{gathered}
T_{1}=\widehat{M}_{0}+\gamma_{3} \sqrt{\sum_{i=1}^{s} \widehat{M}_{i}^{2}}, \quad T_{2}=\frac{\gamma_{s}}{(q+1) !} \sum_{p_{v}=-d}^{r}\left|\delta_{v}-P_{v}\right| M_{q+1}, \\
T_{3}=\left(\sum_{v=1}^{m} \widetilde{b}^{[v]^{\mathrm{T}}} H^{[v] R}+\sum_{v=1}^{m} \widetilde{A}^{[v]} H^{[v] R}\right) g^{[v](s)}(\xi) .
\end{gathered}
$$

It follows from an induction to (3.36) for $n$ that

$$
\left\|\varepsilon_{i}^{(n)}\right\| \leq \begin{cases}\sum_{j=0}^{n}\left(1+2 h \gamma_{4}\right)^{j}\left(T_{1} h^{p+1}+T_{2} h^{q+2}+T_{3} h^{s+2}\right), & i=0, \\ \sum_{j=0}^{n+1}\left(1+2 h \gamma_{4}\right)^{j}\left(T_{1} h^{p+1}+T_{2} h^{q+2}+T_{3} h^{s+2}\right), & i=1,2, \ldots, s .\end{cases}
$$

Hence, for $h \in\left(0, h_{0}\right]$, we arrive at

$$
\begin{aligned}
\left\|y\left(t_{n}\right)-y_{n}\right\| & =\left\|\varepsilon_{0}^{(n)}\right\| \leq \sum_{j=0}^{n}\left(1+2 h \gamma_{4}\right)^{j}\left(T_{1} h^{p+1}+T_{2} h^{q+2}+T_{3} h^{s+2}\right) \\
& =\frac{\left(1+2 h \gamma_{4}\right)^{n+1}-1}{2 h \gamma_{4}}\left(T_{1} h^{p+1}+T_{2} h^{q+2}+T_{3} h^{s+2}\right)
\end{aligned}
$$




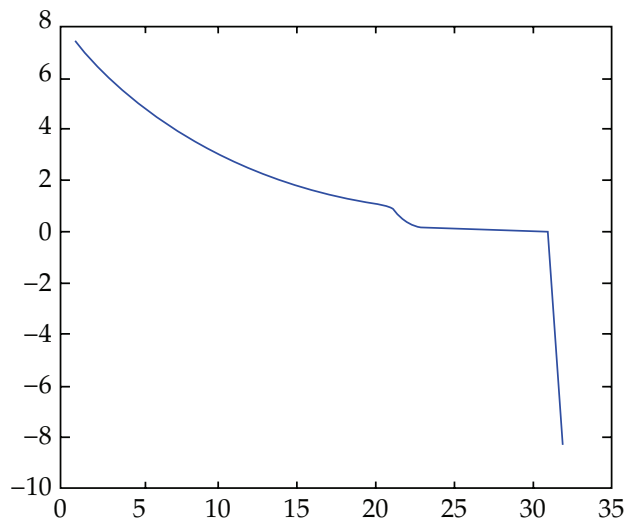

Figure 1: Values $y_{n}$ with $h=0.1$.

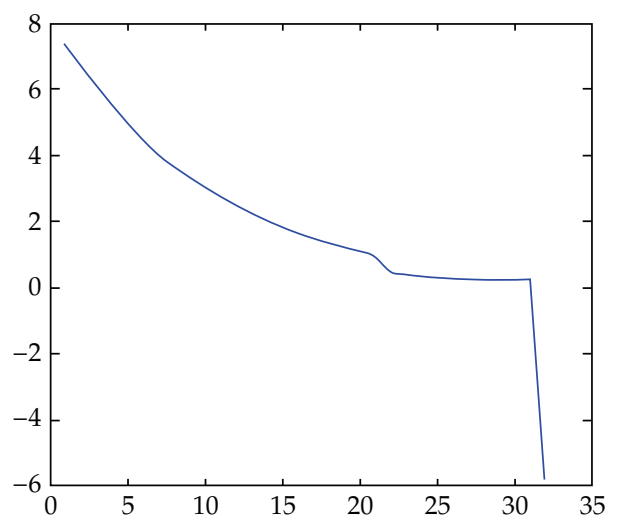

Figure 2: Values $z_{n}$ with $h=0.1$.

$$
\begin{aligned}
& \leq \frac{\exp \left[2(n+1) h \gamma_{4}\right]-1}{2 h \gamma_{4}}\left(T_{1} h^{p+1}+T_{2} h^{q+2}+T_{3} h^{s+2}\right) \\
& \leq \frac{\exp \left[2\left(T-t_{0}\right) \gamma_{4}\right] \exp \left(2 h_{0} \gamma_{4}\right)-1}{2 \gamma_{4}}\left(T_{1} h^{p}+T_{2} h^{q+1}+T_{3} h^{s+1}\right) .
\end{aligned}
$$

Therefore, the ARKLM (2.1) is D-Convergent of order $\min \{p, q+1, s+1\},(q=r+d)$. 
Table 1: Comparison between the numerical solutions for different $h$ and the exact solution.

\begin{tabular}{lcccc}
\hline$t$ & $\begin{array}{c}\text { Numerical solution } \\
\text { for } h=0.2\end{array}$ & $\begin{array}{c}\text { Numerical solution } \\
\text { for } h=0.1\end{array}$ & $\begin{array}{c}\text { Numerical solution } \\
\text { for } h=0.05\end{array}$ & Exact solution $y(t)$ \\
\hline 0.2 & 2.87569414 & 1.212021722 & 0.898712327 & 0.818730753 \\
0.4 & 2.751727517 & 1.165089633 & 0.801604459 & 0.670320046 \\
0.6 & 2.448183694 & 1.0957815 & 0.626189996 & 0.548811636 \\
\hline
\end{tabular}

\section{Some Examples}

Consider the following initial value problem of multidelay-integro-differential equations:

$$
\begin{aligned}
y^{\prime}(t)= & \left(-10^{4}+99 i\right) \frac{[1+y(t)]^{2}}{1+[1+y(t)]^{2}}+500[y(t-1)-y(t-2)]-500 \int_{t-1}^{t} y(s) d s \\
& +500 \int_{t-2}^{t} y(s) d s-\exp (-t)+\left(10^{4}-99 i\right) \times \frac{[1+\exp (-t)]^{2}}{1+[1+\exp (-t)]^{2}}, \quad 0 \leq t \leq 3, \\
y(t)= & \exp (-t), \quad-2 \leq t \leq 0
\end{aligned}
$$

and its perturbed problem:

$$
\begin{aligned}
z^{\prime}(t)= & \left(-10^{4}+99 i\right) \frac{[1+z(t)]^{2}}{1+[1+z(t)]^{2}}+500[z(t-1)-z(t-2)]-500 \int_{t-1}^{t} z(s) d s \\
& +500 \int_{t-2}^{t} z(s) d s-\exp (-t)+\left(10^{4}-99 i\right) \times \frac{[1+\exp (-t)]^{2}}{1+[1+\exp (-t)]^{2}}, \quad 0 \leq t \leq 3, \\
z(t)= & \exp (-t)+0.01, \quad-2 \leq t \leq 0 .
\end{aligned}
$$

It an be easily verified that $a_{1}=a_{2}=-9.5 \times 10^{3}, \beta_{1}=\beta_{2}=250, \sigma_{1}=\sigma_{2}=250$, and $\widetilde{r}_{1}=\widetilde{r}_{2}=1$, with analytic solution $y(t)=\exp (-t)$, where the inner product is standard inner product. We apply the two-stages and two-order additive R-K method:

$$
\begin{array}{l|ll|ll}
1 & 1 & 0 & 1 & 0 \\
1 & \frac{1}{2} & \frac{1}{2} & 0 & 1 \\
\hline & \frac{1}{2} & \frac{1}{2} & 0 & 1
\end{array}
$$


(1) to the problem (4.1) and its perturbed problem (4.2). Since the order of the method is 2 , we adopt the compound trapezoidal rule for computing the integer part. According to the result of Theorems 2.3 and 3.7, the corresponding method for DIDEs is GDN-stable and D-convergent. We denote the numerical solution of problem (4.1) and (4.2) $y_{n}$ and $z_{n}$, where $y_{n}$ and $z_{n}$ are approximations to $y\left(t_{n}\right)$ and $z\left(t_{n}\right)$, respectively. The values $y_{n}$ and $z_{n}$ with $h=0.1$ are listed in Figure 1 , (where the abscissa and ordinate denote variable $n$ and $y_{n}$, resp.) and Figure 2, (where the abscissa and ordinate denote variable $n$ and $z_{n}$, resp.). It is shown in Table 1 that the numerical solutions are toward to the exact solutions as $h \rightarrow 0$.

It is obvious that the corresponding method for MDIDEs is GDN-stable and Dconvergent.

\section{Acknowledgments}

This work was supported by the National Natural Science Foundation of China (11101109) and the Natural Science Foundationof Hei-long-jiang Province of China (A201107).

\section{References}

[1] T. E. Wheldon, J. Kirk, and H. M. Finlay, "Cyclical granulopoiesis in chronic granulocytic leukemia: a simulation study," Blood, vol. 43, no. 3, pp. 379-387, 1974

[2] A. Ruehli, U. Miekkala, A. Bellen, and H. Heeb, "Stable time domain solutions for EMC problems using PEEC circuit models," in Proceedings of the IEEE International Symposium on Electromagnetic Compatibility, pp. 371-376, August 1994.

[3] R. K. Brayton, "Small signal stability criterion for networks containing lossless transmission lines," IBM Journal of Research and Development, vol. 12, pp. 431-440, 1968.

[4] N. Guglielmi, "Inexact Newton methods for the steady state analysis of nonlinear circuits," Mathematical Models E Methods in Applied Sciences, vol. 6, no. 1, pp. 43-57, 1996.

[5] Y. Kuang, Delay Differential Equations with Applications in Population Dynamics, vol. 191 of Mathematics in Science and Engineering, Academic Press Inc., Boston, Mass, USA, 1993.

[6] A. Bellen and M. Zennaro, Numerical Methods for Delay Differential Equations, Numerical Mathematics and Scientific Computation, The Clarendon Press Oxford University Press, New York, NY, USA, 2003.

[7] L. Torelli, "Stability of numerical methods for delay differential equations," Journal of Computational and Applied Mathematics, vol. 25, no. 1, pp. 15-26, 1989.

[8] C. J. Zhang and G. Sun, "Nonlinear stability of Runge-Kutta methods applied to infinite-delaydifferential equations," Mathematical and Computer Modelling, vol. 39, no. 4-5, pp. 495-503, 2004.

[9] A. Bellen and M. Zennaro, "Strong contractivity properties of numerical methods for ordinary and delay differential equations," Applied Numerical Mathematics, vol. 9, no. 3-5, pp. 321-346, 1992.

[10] C.-J. Zhang and S.-F. Li, "Dissipativity and exponentially asymptotic stability of the solutions for nonlinear neutral functional-differential equations," Applied Mathematics and Computation, vol. 119, no. 1, pp. 109-115, 2001.

[11] M. Zennaro, "Contractivity of Runge-Kutta methods with respect to forcing terms," Applied Numerical Mathematics, vol. 11, no. 4, pp. 321-345, 1993.

[12] M. Zennaro, "Asymptotic stability analysis of Runge-Kutta methods for nonlinear systems of delay differential equations," Numerische Mathematik, vol. 77, no. 4, pp. 549-563, 1997.

[13] A. Araújo, "A note on B-stability of splitting methods," Computing and Visualization in Science, vol. 6, no. $2-3$, pp. 53-57, 2004.

[14] B. García-Celayeta, I. Higueras, and T. Roldán, "Contractivity/monotonicity for additive RungeKutta methods: inner product norms," Applied Numerical Mathematics, vol. 56, no. 6, pp. 862-878, 2006.

[15] J. K. Hale and S. M. Verduyn Lunel, Introduction to Functional-Differential Equations, vol. 99 of Applied Mathematical Sciences, Springer, New York, NY, USA, 1993. 
[16] V. Kolmanovskir and A. Myshkis, Applied Theory of Functional-Differential Equations, vol. 85 of Mathematics and its Applications, Kluwer Academic Publishers Group, Dordrecht, The Netherlands, 1992.

[17] Y. Kuang, Delay Differential Equations with Applications in Population Dynamics, vol. 191 of Mathematics in Science and Engineering, Academic Press, Boston, Mass, 1993.

[18] C. Zhang and S. Vandewalle, "General linear methods for Volterra integro-differential equations with memory," SIAM Journal on Scientific Computing, vol. 27, no. 6, pp. 2010-2031, 2006.

[19] C. Zhang and S. Vandewalle, "Stability analysis of Runge-Kutta methods for nonlinear Volterra delayintegro-differential equations," IMA Journal of Numerical Analysis, vol. 24, no. 2, pp. 193-214, 2004.

[20] C. J. Zhang and S. Vandewalle, "Stability criteria for exact and discrete solutions of neutral multidelay-integro-differential equations," Advances in Computational Mathematics, vol. 28, no. 4, pp. 383-399, 2008.

[21] T. Koto, "Stability of $\theta$-methods for delay integro-differential equations," Journal of Computational and Applied Mathematics, vol. 161, no. 2, pp. 393-404, 2003.

[22] C. J. Zhang and Y. Y. He, "The extended one-leg methods for nonlinear neutral delay-integrodifferential equations," Applied Numerical Mathematics, vol. 59, no. 6, pp. 1409-1418, 2009.

[23] T. Koto, "Stability of Runge-Kutta methods for delay integro-differential equations," Journal of Computational and Applied Mathematics, vol. 145, no. 2, pp. 483-492, 2002.

[24] C. Huang and S. Vandewalle, "An analysis of delay-dependent stability for ordinary and partial differential equations with fixed and distributed delays," SIAM Journal on Scientific Computing, vol. 25, no. 5, pp. 1608-1632, 2004.

[25] C. J. Zhang and S. Z. Zhou, "Nonlinear stability and D-convergence of Runge-Kutta methods for delay differential equations," Journal of Computational and Applied Mathematics, vol. 85, no. 2, pp. 225237, 1997.

[26] R. Frank, J. Schneid, and C. W. Ueberhuber, "The concept of -convergence," SIAM Journal on Numerical Analysis, vol. 18, no. 5, pp. 753-780, 1981.

[27] K. Dekker and J. G. Verwer, Stability of Runge-Kutta Methods for Stiff Nonlinear Differential Equations, vol. 2 of CWI Monographs, North-Holland Publishing, Amsterdam, The Netherlands, 1984.

[28] J. D. Lambert, Numerical Methods for Ordinary Differential Systems, John Wiley \& Sons, Chichester, UK, 1991.

[29] W. H. Hundsdorfer, "Stability and B-convergence of linearly implicit Runge-Kutta methods," Numerische Mathematik, vol. 50, no. 1, pp. 83-95, 1986. 


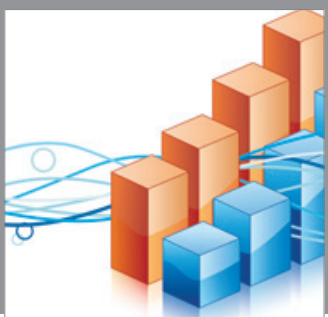

Advances in

Operations Research

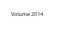

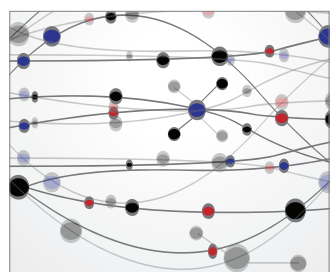

\section{The Scientific} World Journal
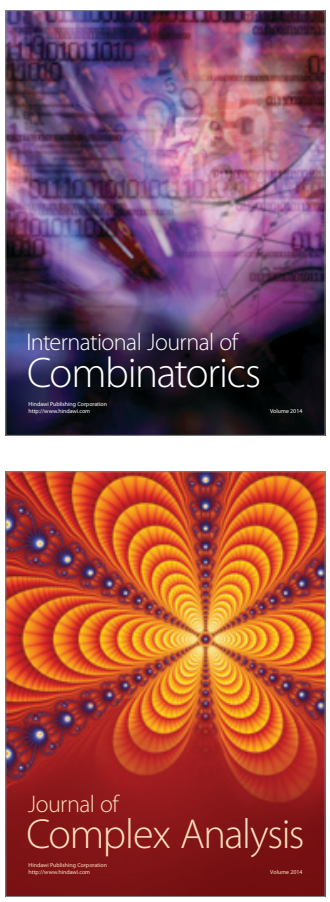

International Journal of

Mathematics and

Mathematical

Sciences
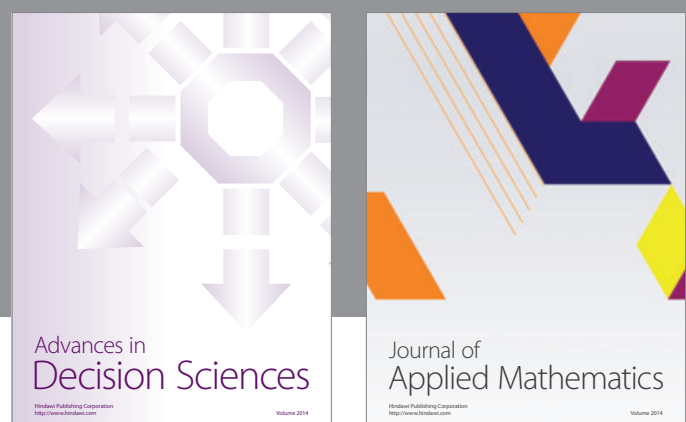

Journal of

Applied Mathematics
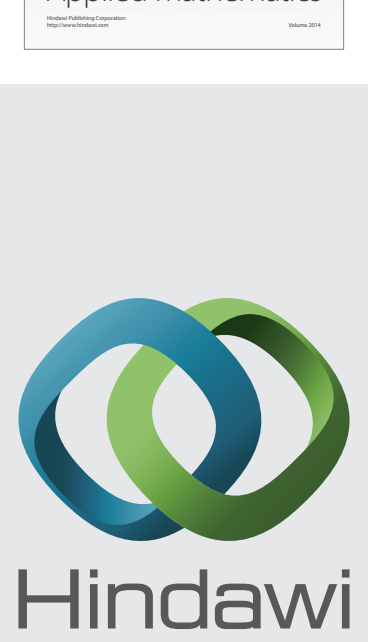

Submit your manuscripts at http://www.hindawi.com
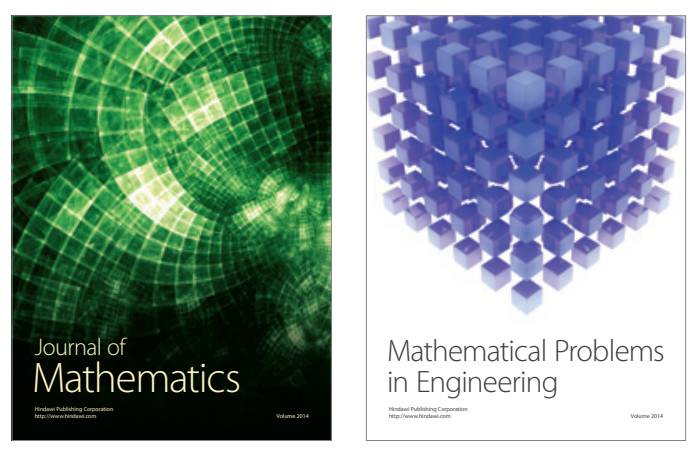

Mathematical Problems in Engineering
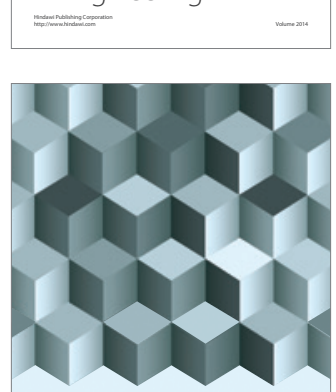

Journal of

Function Spaces
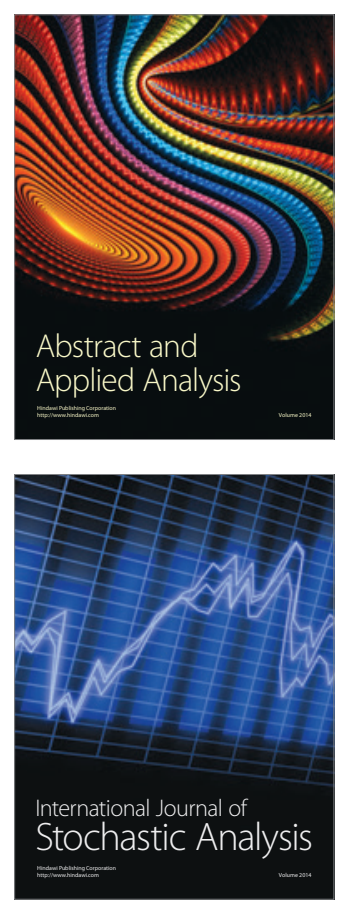

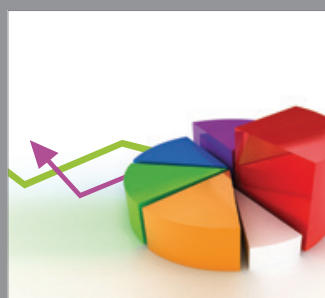

ournal of

Probability and Statistics

Promensencen
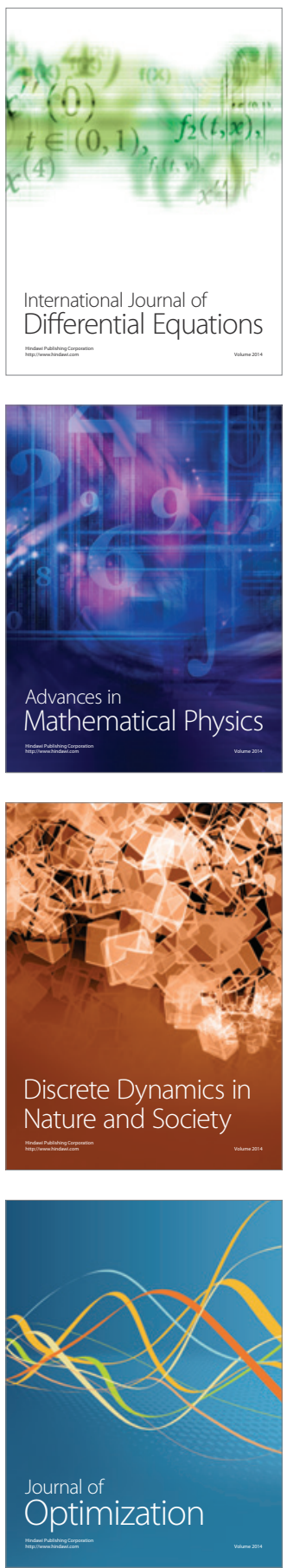